\title{
Pairing Mechanism in the doped Hubbard Antiferromagnet: the $4 \times 4$ Model as a Test Case
}

\author{
Michele Cini, Enrico Perfetto and Gianluca Stefanucci \\ Istituto Nazionale di Fisica della Materia, Dipartimento di Fisica, \\ Universita' di Roma Tor Vergata, Via della Ricerca Scientifica, 1-00133
}

Roma, Italy

\begin{abstract}
We introduce a local formalism, in terms of eigenstates of number operators, having well defined point symmetry, to solve the Hubbard model at weak coupling on a $N \times N$ square lattice (for even $N$ ). The key concept is that of $W=0$ states, that are the many-body eigenstates of the kinetic energy with vanishing Hubbard repulsion. At half filling, the wave function demonstrates an antiferromagnetic order, a lattice step translation being equivalent to a spin flip. Further, we state a general theorem which allows to find all the $W=0$ pairs (two-body $W=0$ singlet states). We show that, in special cases, this assigns the ground state symmetries at least in the weak coupling regime. The $N=4$ case is discussed in detail. To study the doped half filled system, we enhance the Group Theory analysis of the $4 \times 4$ Hubbard model introducing an Optimal Group which explains all the degeneracies in the one-body and many-body spectra. We use the Optimal Group to predict the possible ground state symmetries of the $4 \times 4$ doped antiferromagnet by means of our general theorem and the results are in agreement with exact diagonalization data. Then we create $W=0$ electron pairs over the antiferromagnetic state. We show analitycally that the effective interaction between the electrons of the pairs is attractive and forms bound states. Computing the corresponding binding energy we are able to definitely predict the exact ground state symmetry.
\end{abstract}

\section{INTRODUCTION}

The (repulsive) $2 D$ Hubbard hamiltonian is one of the popular models of the high- $T_{C}$ cuprates [1], as many people believe that it contains at least some of the relevant ingredients of the mechanism of superconductivity. While other ingredients may well be missing for the full explanation of superconductivity and the rich phase diagram of these materials, there are now strong evidences of pairing in this model, and although pairing is not synonimous to superconductivity it can hardly be supposed to be extraneous to it. The evidence for pairing comes from various independent methods, including cluster diagonalizations [2] [3] [4], fluctuation exchange (FLEX) [5] diagrammatic approach, which is based on a conserving approximation, and renormalization group techniques [6] [7]. The approach we are proposing is based on an analytic canonical transformation, reminiscent of the original Cooper theory. However, our understanding of the many-body ground state is only partial, and the symmetries of the bound pairs have not yet been fully explored. The Hubbard model must be more thoroughly understood before we can solve more realistic ones.

In the strong coupling limit the double occupation of the same site is energetically suppressed and the model at half filling is equivalent to the Heisenberg model with an antiferromagnetic exchange interaction [8]. A popular approach takes care of the strong repulsion between two opposite spin fermions by a Gutzwiller [9] projection, i.e. by throwing out of the Hilbert space the double occupation states. However, truncating the Hilbert space in this way costs kinetic energy, so at finite $U$ the system must allow double occupation, also in the ground state, as one can see from the eigenvectors of cluster calculations. At weak coupling, on the other hand, it makes sense to speak about particles in filled shells, which behave much as core electrons in atomic physics, and particles in partially filled, or valence, shells. Remarkably, particles in partially filled shells can totally avoid double occupation at

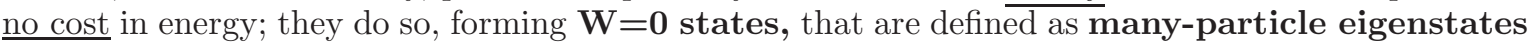
of the kinetic energy with no double occupation. Below, using a new formalism, we show how $W=0$ states arise by symmetry. We stress that since $W=0$ states emerge from symmetry alone, they remain $W=0$ for any coupling strength and are an adequate starting point for a realistic theory. This is the reason why weak coupling expansions often provide good approximations at intermediate coupling, as observed by several Authors [10] [11] [12]. 


\section{A. Canonical Transformation Approach to the Pairing Mechanism}

Let us consider the Hubbard model with hamiltonian

$$
H=H_{0}+W=t \sum_{\sigma} \sum_{\left\langle r, r^{\prime}\right\rangle} c_{r \sigma}^{\dagger} c_{r^{\prime} \sigma}+\sum_{r} U \hat{n}_{r \uparrow} \hat{n}_{r \downarrow}, \quad U>0,
$$

on a square lattice of $N \times N$ sites with periodic boundary conditions and even $N$. Here $\sigma=\uparrow, \downarrow$ is the spin and $r, r^{\prime}$ the spatial degrees of freedom of the hole creation and annihilation operators $c^{\dagger}$ and $c$ respectively. The sum on $\left\langle r, r^{\prime}\right\rangle$ is over the pairs of nearest neighbors sites and $\hat{n}_{r \sigma}$ is the number operator on the site $r$ of spin $\sigma$. The point symmetry is $C_{4 v}$, the Group of a square [13]; besides, $H$ is invariant under the commutative Group of Translations $\mathbf{T}$ and hence the Space Group [14] $\mathbf{G}=\mathbf{T} \otimes C_{4 v} ; \otimes$ means the semidirect product. We represent sites by $r=\left(i_{x}, i_{y}\right)$ and wave vectors by $k=\left(k_{x}, k_{y}\right)=\frac{2 \pi}{N}\left(i_{x}, i_{y}\right)$, with $i_{x}, i_{y}=0, \ldots, N-1$. In terms of the Fourier expanded fermion operators $c_{k \sigma}=\frac{1}{N} \sum_{r} e^{i k r} c_{r \sigma}$, we have $H_{0}=\sum_{k} \epsilon(k) c_{k \sigma}^{\dagger} c_{k \sigma}$ with $\epsilon(k)=2 t\left[\cos k_{x}+\cos k_{y}\right]$. Then the one-body plane wave state $c_{k \sigma}^{\dagger}|0\rangle \equiv|k \sigma\rangle$ is an eigenstate of $H_{0}$.

To study the behaviour of two holes added to the system in its ground state, we introduce the $W=0$ pairs, a special case of $W=0$ states. Using degenerate eigenstates of the kinetic energy $H_{0}$, the non-abelian point symmetry Group $C_{4 v}$ of the Hubbard hamiltonian (1) allows the existence of two-body singlet states with no double occupancy. They are obtained by a configuration interaction mechanism and may be of special interest since the particles of a $W=0$ pair do not feel any direct repulsion. Hence a new pairing scenario arises: since the two extra particles cannot interact directly, by definition of $W=0$ pair, their effective interaction comes out from virtual electron-hole excitations exchanges with the Fermi sea and in priciple can be attractive. In the following we show how to get the effective interaction between two holes added to the system.

Many configurations contribute to the interacting $(n+2)$-body ground state $\left|\Psi_{0}(n+2)\right\rangle$ and we need a complete set $\mathcal{S}$ to expand it exactly; as long as it is complete, however, we can $\operatorname{design} \mathcal{S}$ as we please. We can take the non-interacting $n$-body Fermi sphere $|\Phi(n)\rangle$ as our vacuum and build the complete set in terms of excitations over the vacuum. In the subspace with vanishing spin $z$ component, the simplest states that enter the configuration mixing are those obtained from $|\Phi(n)\rangle$ by creating two extra holes over it (we call them the $m$ states); the set

$$
c_{k_{1} \uparrow}^{\dagger} c_{k_{2} \downarrow}^{\dagger}|\Phi(n)\rangle, \quad \epsilon\left(k_{1}\right), \epsilon\left(k_{2}\right)>\epsilon_{F}
$$

where $\epsilon_{F}$ is the Fermi energy, is a basis for this part of the Hilbert space. Similarly, along with the pair $m$ states, we introduce the 4-body $\alpha$ states, obtained from $|\Phi(n)\rangle$ by creating 2 holes and 1 electron-hole (e-h) pair; a basis looks like

$$
c_{k_{1} \uparrow}^{\dagger} c_{k_{2} \downarrow}^{\dagger} c_{k_{3} \sigma} c_{k_{4} \sigma}^{\dagger}|\Phi(n)\rangle, \quad \epsilon\left(k_{1}\right), \epsilon\left(k_{2}\right), \epsilon\left(k_{4}\right)>\epsilon_{F}>\epsilon\left(k_{3}\right), \quad \sigma=\uparrow, \downarrow
$$

Then $\mathcal{S}$ includes the 6 -body $\beta$ states having 2 holes and 2 e-h pairs, and so on until states with two holes and $n$ e-h pairs if we are below half filling or $2 N^{2}-n$ e-h pairs if we are above. We are using Greek indices for the configurations containing the electron-hole pairs, which here are playing largely the same rôle as phonons in the Cooper theory. By means of the complet set $\mathcal{S}$ we now expand the interacting ground state

$$
\left|\Psi_{0}(n+2)\right\rangle=\sum_{m} a_{m}|m\rangle+\sum_{\alpha} b_{\alpha}|\alpha\rangle+\sum_{\beta} c_{\beta}|\beta\rangle+\ldots
$$

and set up the Schrödinger equation

$$
H\left|\Psi_{0}(n+2)\right\rangle=E\left|\Psi_{0}(n+2)\right\rangle .
$$

We stress that Eq.(4) is configuration interaction, not a perturbative expansion.

When the number $n$ of holes in the $N \times N$ system is such that $|\Phi(n)\rangle$ is a single non-degenerate determinant (the Fermi surface is totally filled), we can easily and unambiguously define and calculate the effective interaction between the two extra holes since the expansion (4) for the interacting 
ground state is unique: this is done by a canonical transformation [15], [16], [17] from the many-body Hamiltonian of Eq.(1). We consider the effects of the operators on the terms of $\left|\Psi_{0}(n+2)\right\rangle$. We write:

$$
H_{0}|m\rangle=E_{m}|m\rangle,
$$

and since $W$ can create or destroy up to 2 e-h pairs, its action on an $m$ state yields

$$
W|m\rangle=\sum_{m^{\prime}} W_{m^{\prime}, m}\left|m^{\prime}\right\rangle+\sum_{\alpha}|\alpha\rangle W_{\alpha, m}+\sum_{\beta}|\beta\rangle W_{\beta, m} .
$$

The matrix elements of the two-body interaction $W$ between determinants which differ by two spinorbitals reduces to a bielectronic integral. Hence one can show that if $m^{\prime}, m$ represent different $W=0$ pairs added to the vacuum, $W_{m^{\prime}, m}$ vanishes. Moreover,

$$
W|\alpha\rangle=\sum_{m}|m\rangle W_{m, \alpha}+\sum_{\alpha^{\prime}}\left|\alpha^{\prime}\right\rangle W_{\alpha^{\prime}, \alpha}+\sum_{\beta}|\beta\rangle W_{\beta, \alpha}+\sum_{\gamma}|\gamma\rangle W_{\gamma \alpha},
$$

where scattering between 4-body states is allowed by the second term, and so on. In this way we obtain an algebric system for the coefficients of the configuration interaction (4). However to test the instability of the Fermi liquid towards pairing it is sufficient to study the amplitudes $a_{m}$ of the $m$ states. In the weak coupling limit this can be done truncating the expansion (4) to the $\alpha$ states because, as we have shown [15], the inclusion of the $\beta, \gamma, \ldots$ states produces a renormalization of the matrix elements of higher order in $W$, leaving the structure of the equations unaltered. Choosing the $\alpha$ states such that

$$
\left(H_{0}+W\right)_{\alpha \alpha^{\prime}}=\delta_{\alpha \alpha^{\prime}} E_{\alpha}^{\prime}
$$

the algebric system reduces to

$$
\begin{gathered}
\left(E_{m}-E\right) a_{m}+\sum_{m^{\prime}} a_{m^{\prime}} W_{m, m^{\prime}}+\sum_{\alpha} b_{\alpha} W_{m, \alpha}=0 \\
\left(E_{\alpha}^{\prime}-E\right) b_{\alpha}+\sum_{m^{\prime}} a_{m^{\prime}} W_{\alpha, m^{\prime}}=0 .
\end{gathered}
$$

Solving for $b_{\alpha}$ and substituting in the first equation we exactly decouple the 4-body states as well, ending up with an equation for the dressed pair $|a\rangle=\sum_{m} a_{m}|m\rangle$. The effective Schrödinger equation for the pair reads

$$
\left(H_{0}+W+W_{e f f}[E]\right)|a\rangle=E|a\rangle
$$

where

$$
\left(W_{e f f}\right)_{m m^{\prime}}=-\sum_{\alpha} \frac{W_{m \alpha} W_{\alpha m^{\prime}}}{E_{\alpha}^{\prime}-E} .
$$

Hence Eq.(12) is a self-consistent equation and we have calculated the effective interaction $W_{\text {eff }}$ between $W=0$ pairs analytically [16], [17]; it can be attractive depending on $n$. Also we have found that the results compare well with exact diagonalization results, when available. Basically the same mechanism works for small clusters with open boundary conditions [3].

We want to stress that the truncated expansion of $\left|\Psi_{0}(n+2)\right\rangle$ in Eq.(4) doesn't give a good approximation of the interacting ground state wave function but only of its weak coupling $a_{m}$ amplitudes. Similarly in the BCS model, from the Cooper equation (obtained by truncation of $\left|\Psi_{0}(n+2)\right\rangle$ to the $m$ states) we can estimate only the pair coefficients of the ground state and not its full structure. Nevertheless this is enough to study bound states formation; indeed the energy gap of the pair in the Cooper theory and in the many-body BCS theory are equals. 


\section{B. Truncated Configuration Interaction: Description of the Pairing Mechanism in Small Clusters}

Although the above canonical transformation can be performed without any truncation of the configuration interaction expansion, the description simplifies if we take into account $\alpha$ states only. As observed above this is justified in the weak coupling regime and is enough to study the pairing problem. Let us consider for example the pairing mechanism in small clusters, like $\mathrm{CuO}_{4}$ and $\mathrm{Cu} \mathrm{O}_{5} \mathrm{O}_{16}$. Such clusters with 4 holes have ground states of ${ }^{1} B_{2}$ symmetry in $C_{4 v}$ for $0<U \leq t$. The diagnosis that hole pairing between the holes of a $W=0$ pair occurs in these ground states results from the following steps.

a) The lowest eigenstate of the one-body energy spectrum has $A_{1}$ symmetry and the interacting ground state with two holes is a $C_{4 v}$ totalsymmetric singlet. The state $|\Phi\rangle=c_{A_{1} \uparrow}^{\dagger} c_{A_{1} \downarrow}^{\dagger}|0\rangle$ is the twobody $U=0$ non-degenerate ground state, where here and in the following steps $c_{I \sigma}^{\dagger}$ is the creation operator for the hole eigenstate of $I$ symmetry and spin $\sigma$. b) The first excited level of the one-body energy spectrum is degenerate and the corresponding eigenstates transform as the vector components $(x, y) . c)$ To have a ground state with four holes of ${ }^{1} B_{2}$ symmetry in the limit of vanishing interaction we must create two holes over $|\Phi\rangle$ in the singlet state $\left|m_{S}\right\rangle \equiv \frac{1}{\sqrt{2}}\left(c_{x \uparrow}^{\dagger} c_{y \downarrow}^{\dagger}+c_{y \uparrow}^{\dagger} c_{x \downarrow}^{\dagger}\right)|\Phi\rangle$. $\left.d\right)$ Taking $\left|m_{S}\right\rangle$ as our unique $m$ state we apply the above canonical transformation and we find an effective attractive interaction summing over all the virtual 4-body (3 holes-1 electron) intermediate states.

As another description to this weak-coupling case, we may say we are doing degenerate second-order perturbation theory; in this example, the zero-th approximation eigenstates are just two, $\left|m_{1}\right\rangle \equiv$ $c_{x \uparrow}^{\dagger} c_{y \downarrow}^{\dagger}|\Phi\rangle$ and $\left|m_{2}\right\rangle \equiv c_{y \uparrow}^{\dagger} c_{x \downarrow}^{\dagger}|\Phi\rangle$, and the problem is reduced to a $2 \times 2$ matrix diagonalization. The corresponding second-order interaction is illustrated in Figure I: it takes the state $\left|m_{1}\right\rangle$ into $\left|m_{2}\right\rangle$ and conversely. Hence it is actually a spin-flip interaction.

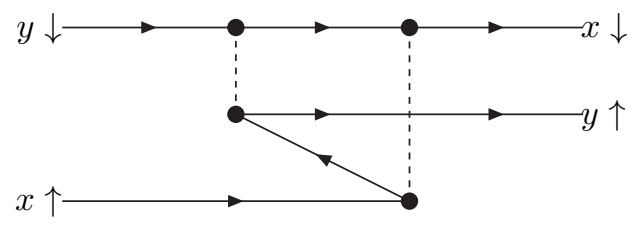

Figure I. The e-h exchange diagram for the Two-Hole Amplitude. For $W=0$ pairs, the direct interaction vanishes and this diagram produces an effective interaction, splitting singlet and triplet pairs.

It turns out [3] that the effective interaction is attractive in the singlet $\left|\Psi_{1_{1}}\right\rangle \equiv\left|m_{S}\right\rangle=\frac{1}{\sqrt{2}}\left(\left|m_{1}\right\rangle+\right.$ $\left.\left|m_{2}\right\rangle\right)$ and equal in size, but repulsive, in the triplet $\left|\Psi_{3_{B}}\right\rangle \equiv\left|m_{T}\right\rangle=\frac{1}{\sqrt{2}}\left(\left|m_{1}\right\rangle-\left|m_{2}\right\rangle\right)$. That is definitely a pairing situation for the singlet. e) The two-body state $\frac{1}{\sqrt{2}}\left(c_{x \uparrow}^{\dagger} c_{y \downarrow}^{\dagger}+c_{y \uparrow}^{\dagger} c_{x \downarrow}^{\dagger}\right)|0\rangle$ is a $W=0$ pair (no direct interaction occurs between the holes); hence we can say that the $W=0$ pair, when dressed by virtual e-h excitations, gives up a bound pair.

These are the principles that here we wish to extend to the half filled $4 \times 4$ Hubbard model of Eq.(1).

\section{Plan of the paper}

Below, we consider the ground state of the $4 \times 4$ cluster with 14,15 and 16 holes, and demonstrate that the 14 hole case must be interpreted in terms of pairing of two electrons added to the half-filled antiferromagnet. However the non-interacting ground state is degenerate and this means that we must extend the above formalism.

The physical interpretation emerges from the fact that we are able to solve the problem analytically at weak coupling. When the analytic results are compared to the numerical ones [2] we can conclude 
that $i$ ) we are able to predict from Group Theory the good quantum numbers and degeneracies of the ground states involved at various occupancies $i i)$ we are able to show that the effective interaction between the extra electrons is attractive in the singlet (ground state) and repulsive for the triplet.

These analytic results have been allowed by a deeper symmetry analysis of the $4 \times 4$ cluster than had been possible previously. The antiferromagnetic ground state has also been explored analytically by a new approach. In this way, the electron pair creation is also accoplished analytically, with full control of the symmetry.

Due to the special role played by $W=0$ pairs, in the next Section we state a general theorem to obtain all the possible $W=0$ pairs. The theorem requires the knowledge of a (previously unknown) symmetry Group big enough to explain the one-body degeneracies (Optimal Group). As we shall see the Space Group G does not work and in Section III we determine the Optimal Group for the $4 \times 4$ square lattice. In Section IV we determine the exact weak coupling ground state wave function at half filling, that is unique as granted by the Lieb's theorem [18]. This will be done using a new local formalism that enables us to write down all the $W=0$ states of the partially filled shell. In Section $\mathrm{V}$, with the help of the theorem of Section II and with the Optimal Group in hands, we single out all $W=0$ pairs formed from degenerate orbitals of the shell $\epsilon(k)=0$. Hence in Section VI we use the corresponding local basis to write down $W=0$ two and four-body states. Then to study the pairing problem at half filling we extend the above canonical transformation and finally in Section VII we shall reduce the pairing problem to $2 \times 2$ matrices and compare with the literature numerical data. Finally, we underline the implications of the present results in Section VIII.

\section{W=0 PAIRS AND SYMMETRIES OF THE DOPED GROUND STATES: A USEFUL THEOREM}

In general, one has a set $\mathcal{S}$ of degenerate eigenstates of $H_{0}$ which is partially filled in the $U=0$ limit. To first-order, we ignore the particles in the filled shells and find the exact ground state(s) of $W$ in the truncated Hilbert space $\mathcal{H}$ describing those in the partially filled shell. One simple case occurs when only two holes are in the partially filled shell, because the ground states are $W=0$ pairs (filled shells are understood). We recall that they are two body singlets that are eigenstates of $H_{0}$ and belong to the kernel of $W$. There is no double occupation of any site in such a pair. This means that the two particles of a $W=0$ pair do not interact directly, but only by means of virtual electron-hole excitations.

As we shall see, another special case occurs when the system at half filling is doped by removing two particles (they can only be removed as $W=0$ pairs).

In this Section we want to show how $W=0$ pairs arise, due to the symmetry of the system. Previously [15] [16] we have shown that $W=0$ pairs with zero total momentum are a consequence of the $C_{4 v}$ point symmetry. Projecting the determinantal state

$$
|d(k)\rangle=c_{k \uparrow}^{\dagger} c_{-k \downarrow}^{\dagger}|0\rangle
$$

on the irreducible representations $A_{2}, B_{1}$ and $B_{2}$ of $C_{4 v}$ one obtains $W=0$ pairs.

Here we want to point out a more powerful and elegant criterion to get all the $W=0$ pairs, including those of nonvanishing total momentum. We can do that in terms of the Optimal Group $\mathcal{G}$ of the Hamiltonian, that we define as a symmetry Group which is big enough to justify the degeneracy of the single particle energy levels. By definition, every one-body eigenstate of $H$ can be classified as belonging to one of the irreps of $\mathcal{G}$. We may say that an irrep $\eta$ is represented in the one-body spectrum of $H$ if at least one of the one-body levels belongs to $\eta$. Let $\mathcal{E}$ be the set of the irreps of $\mathcal{G}$ which are represented in the one-body spectrum of $H$. Let $|\psi\rangle$ be a two-body eigenstate of the kinetic energy $H_{0}$ with spin $S_{z}=0$ and $P^{(\eta)}$ the projection operator on the irrep $\eta$. We wish to prove the

$W=0$ Theorem:

$$
\eta \notin \mathcal{E} \Leftrightarrow W P^{(\eta)}|\psi\rangle=0
$$

In other terms, any nonvanishing projection of $|\psi\rangle$ on an irrep not contained in $\mathcal{E}$, is an eigenstate of $H_{0}$ with no double occupancy. The singlet component of this state is a $W=0$ pair. Conversely, any pair belonging to an irrep represented in the spectrum must have positive $W$ expectation value. 
In the case of the $4 \times 4$ model (see Section V) the pairs belonging to irreps of the Optimal Group have well defined parities under particle exchange, and can be classified as singlet or triplet pairs.

Proof: Let us consider a two body state of opposite spins trasforming as the $i$-th component of the irrep $\eta$ of $\mathcal{G}$ :

$$
\left|\psi_{i}^{(\eta)}\right\rangle=\sum_{r_{1} r_{2}} \psi_{i}^{(\eta)}\left(r_{1}, r_{2}\right) c_{r_{1} \uparrow}^{\dagger} c_{r_{2} \downarrow}^{\dagger}|0\rangle
$$

Then we have

$$
\hat{n}_{r \uparrow} \hat{n}_{r \downarrow}\left|\psi_{i}^{(\eta)}\right\rangle=\psi_{i}^{(\eta)}(r, r) c_{r \uparrow}^{\dagger} c_{r \downarrow}^{\dagger}|0\rangle \equiv \psi_{i}^{(\eta)}(r, r)|r \uparrow, r \downarrow\rangle .
$$

We define $P_{i}^{(\eta)}$ as the projection operator on the $i$-th component of the irrep $\eta$. Since

$$
P_{i}^{(\eta)} \sum_{r} \psi_{i}^{(\eta)}(r, r)|r \uparrow, r \downarrow\rangle=\sum_{r} \psi_{i}^{(\eta)}(r, r)|r \uparrow, r \downarrow\rangle
$$

if

$$
P_{i}^{(\eta)}|r \uparrow, r \downarrow\rangle=0 \quad \forall r
$$

then $\psi_{i}^{(\eta)}(r, r)=0 \forall r$. It is worth to note that Eq.(19) is true if and only if

$$
P_{i}^{(\eta)}|r \sigma\rangle=0 \quad \forall r
$$

where $|r \sigma\rangle=c_{r \sigma}^{\dagger}|0\rangle$.

It is always possible to write $|r \sigma\rangle$ as

$$
|r \sigma\rangle=\sum_{\eta \in \mathcal{E}} \sum_{i} c_{i}^{(\eta)}(r)\left|\varphi_{i, \sigma}^{(\eta)}\right\rangle
$$

where $\left|\varphi_{i, \sigma}^{(\eta)}\right\rangle$ is the one-body eigenstate of $H_{0}$ with spin $\sigma$ transforming as the $i$-th component of the irrep $\eta$. From (20) it follows directly that if $\eta^{\prime}$ does not belong to $\mathcal{E}$

$$
P^{\left(\eta^{\prime}\right)}|r \sigma\rangle=0
$$

and so $P^{\left(\eta^{\prime}\right)}|r \uparrow, r \downarrow\rangle=0$.

This theorem restricts the possible ground state symmetries in some special case. Let $U_{c}(n)$ be the minimum crossover value of $U$, that is the ground state with $n$ holes $\left|\Psi_{0}(n)\right\rangle$ has well defined symmetry $\eta_{0}$ for $0<U<U_{c}(n)$. Let $|\Phi(n)\rangle$ be non-degenerate (closed shells case). Then if we add two extra holes, the new ground state for $U=0$ is a $W=0$ pair over $|\Phi(n)\rangle$, and its symmetry is $\eta_{0} \cdot \eta_{W=0}$, where $\eta_{W=0}$ is the symmetry of the added pair. Turning on the interaction the symmetry cannot change if $U<U_{c}(n+2)$ ! This restriction is posed by Group theory alone: which of the symmetries that remain allowed is actually realised in the ground state depends on the dynamics.

The complete characterization of the symmetry of $W=0$ pairs requires the knowledge of the Optimal Group $\mathcal{G}$. A partial use of the theorem is possible if one does not know $\mathcal{G}$ but knows a subgroup, like the Space Group G. It is then still granted that any pair belonging to an irrep of $\mathbf{G}$ not represented in the spectrum has the $W=0$ property. On the other hand, accidental degeneracies occur with a subgroup of the Optimal Group, and by mixing degenerate pairs belonging to irreps represented in the spectrum one can find $W=0$ pairs also there. This is clearly illustrated by the example repported in the next Section.

\section{THE OPTIMAL GROUP FOR THE $4 \times 4$ HUBBARD MODEL}

The half filled shell of the $4 \times 4$ Hubbard model has degeneracy 6, and the Space Group G does not have irreps with dimensions bigger than 4 [14]. An additional symmetry is needed to justify the sixfold degenerate eigenvalue $\epsilon(k)=0$, and we have found it [19]. Let us represent the $4 \times 4$ lattice as 


\begin{tabular}{|c|c|c|c|}
\hline 1 & 2 & 3 & 4 \\
\hline 5 & 6 & 7 & 8 \\
\hline 9 & 10 & 11 & 12 \\
\hline 13 & 14 & 15 & 16 \\
\hline
\end{tabular}

Periodic boundary conditions are assumed and for example, the nearest neighbours of 1 are $2,5,4$ and 13. Rotating the plaquettes $1,2,5,6$ and 11,12,15,16 clockwise and the other two counterclockwise by 90 degrees we obtain the effect of the "dynamical" symmetry, that we call $d$ :

\begin{tabular}{|c|c|c|c|}
\hline 5 & 1 & 4 & 8 \\
\hline 6 & 2 & 3 & 7 \\
\hline 10 & 14 & 15 & 11 \\
\hline 9 & 13 & 16 & 12 \\
\hline
\end{tabular}

This transformation preserves nearest neighbours (and so, each order of neighbours) but is not an isometry, and for example the distance between 1 and 3 changes. Thus, this symmetry operation $d$ is a new, dynamical symmetry. Including $d$ and closing the multiplication table we obtain the Optimal Group $\mathcal{G}$ with 384 elements in 20 classes (like $\mathbf{G}$ ) as shown in Table I.

\begin{tabular}{|c|c|c|c|c|c|c|c|c|c|}
\hline $\mathcal{C}_{1}$ & $\mathcal{C}_{2}$ & $\mathcal{C}_{3}$ & $\mathcal{C}_{4}$ & $\mathcal{C}_{5}$ & $\mathcal{C}_{6}$ & $\mathcal{C}_{7}$ & $\mathcal{C}_{8}$ & $\mathcal{C}_{9}$ & $\mathcal{C}_{10}$ \\
\hline$I$ & $t_{22}$ & $C_{4} \sigma^{\prime}[2]$ & $\sigma_{x}$ & $C_{2}$ & $\sigma^{\prime}$ & $C_{2} d$ & $C_{2} t_{22} d$ & $C_{2} \sigma^{\prime}[1]$ & $C_{4}^{3} t_{02} d$ \\
\hline $\mathcal{C}_{11}$ & $\mathcal{C}_{12}$ & $\mathcal{C}_{13}$ & $\mathcal{C}_{14}$ & $\mathcal{C}_{15}$ & $\mathcal{C}_{16}$ & $\mathcal{C}_{17}$ & $\mathcal{C}_{18}$ & $\mathcal{C}_{19}$ & $\mathcal{C}_{20}$ \\
\hline$C_{4}^{3} t_{20} d$ & $C_{2} t_{01}$ & $C_{2} \sigma_{x}[1]$ & $C_{4}$ & $C_{2} t_{01} d$ & $C_{2} t_{12} d$ & $C_{2} \sigma_{x}[1] d$ & $C_{4}[1] d$ & $C_{2}[1] d$ & $C_{4}[1]$ \\
\hline
\end{tabular}

Table I. Here, we report one operation for each of the 20 classes $\mathcal{C}_{i}$; the others can be obtained by conjugation. The operations are: the identity $I$, the translation $t_{m n}$ of $m$ steps along $x$ and $n$ along $y$ axis; the other operations $C_{2}, C_{4}, \sigma, \sigma^{\prime}$ are those of the Group of the square and are referenced to the centre; however, $C_{2}[i], C_{4}[i], \sigma[i]$, and $\sigma^{\prime}[i]$ are centered on site $i$.

The complete Character Table of $\mathcal{G}$ is shown as Table II. 


\begin{tabular}{|c|c|c|c|c|c|c|c|c|c|c|c|c|c|c|c|c|c|c|c|c|}
\hline $\mathcal{G}$ & $\mathcal{C}_{1}$ & $\mathcal{C}_{2}$ & $\mathcal{C}_{3}$ & $\mathcal{C}_{4}$ & $\mathcal{C}_{5}$ & $\mathcal{C}_{6}$ & $\mathcal{C}_{7}$ & $\mathcal{C}_{8}$ & $\mathcal{C}_{9}$ & $\mathcal{C}_{10}$ & $\mathcal{C}_{11}$ & $\mathcal{C}_{12}$ & $\mathcal{C}_{13}$ & $\mathcal{C}_{14}$ & $\mathcal{C}_{15}$ & $\mathcal{C}_{16}$ & $\mathcal{C}_{17}$ & $\mathcal{C}_{18}$ & $\mathcal{C}_{19}$ & $\mathcal{C}_{20}$ \\
\hline$A_{1}$ & 1 & 1 & 1 & 1 & 1 & 1 & 1 & 1 & 1 & 1 & 1 & 1 & 1 & 1 & 1 & 1 & 1 & 1 & 1 & 1 \\
\hline$\tilde{A}_{1}$ & 1 & 1 & 1 & 1 & 1 & 1 & -1 & -1 & 1 & -1 & -1 & -1 & -1 & 1 & 1 & 1 & 1 & 1 & -1 & -1 \\
\hline$B_{2}$ & 1 & 1 & -1 & -1 & 1 & 1 & 1 & 1 & 1 & -1 & -1 & 1 & -1 & -1 & 1 & 1 & -1 & -1 & 1 & -1 \\
\hline$B_{2}$ & 1 & 1 & -1 & -1 & 1 & 1 & -1 & -1 & 1 & 1 & 1 & -1 & 1 & -1 & 1 & 1 & -1 & -1 & -1 & 1 \\
\hline$\Gamma_{1}$ & 2 & 2 & -2 & -2 & 2 & 2 & 0 & 0 & 2 & 0 & 0 & 0 & 0 & -2 & -1 & -1 & 1 & 1 & 0 & 0 \\
\hline$\Gamma_{2}$ & 2 & 2 & 2 & 2 & 2 & 2 & 0 & 0 & 2 & 0 & 0 & 0 & 0 & 2 & -1 & -1 & -1 & -1 & 0 & 0 \\
\hline$\Sigma_{1}$ & 3 & 3 & 3 & 3 & 3 & -1 & -1 & -1 & -1 & -1 & -1 & -1 & -1 & -1 & 0 & 0 & 0 & 0 & 1 & 1 \\
\hline$\Sigma_{2}$ & 3 & 3 & 3 & 3 & 3 & -1 & 1 & 1 & -1 & 1 & 1 & 1 & 1 & -1 & 0 & 0 & 0 & 0 & -1 & -1 \\
\hline$\Sigma_{3}$ & 3 & 3 & -3 & -3 & 3 & -1 & -1 & -1 & -1 & 1 & 1 & -1 & 1 & 1 & 0 & 0 & 0 & 0 & 1 & -1 \\
\hline$\Sigma_{4}$ & 3 & 3 & -3 & -3 & 3 & -1 & 1 & 1 & -1 & -1 & -1 & 1 & -1 & 1 & 0 & 0 & 0 & 0 & -1 & 1 \\
\hline$\Lambda_{1}$ & 4 & -4 & -2 & 2 & 0 & 0 & -2 & 2 & 0 & -2 & 2 & 0 & 0 & 0 & -1 & 1 & -1 & 1 & 0 & 0 \\
\hline$\Lambda_{2}$ & 4 & -4 & -2 & 2 & 0 & 0 & 2 & -2 & 0 & 2 & -2 & 0 & 0 & 0 & -1 & 1 & -1 & 1 & 0 & 0 \\
\hline$\Lambda_{3}$ & 4 & -4 & 2 & -2 & 0 & 0 & -2 & 2 & 0 & 2 & -2 & 0 & 0 & 0 & -1 & 1 & 1 & -1 & 0 & 0 \\
\hline$\Lambda_{4}$ & 4 & -4 & 2 & -2 & 0 & 0 & 2 & -2 & 0 & -2 & 2 & 0 & 0 & 0 & -1 & 1 & 1 & -1 & 0 & 0 \\
\hline$\Omega_{1}$ & 6 & 6 & 0 & 0 & -2 & -2 & -2 & -2 & 2 & 0 & 0 & 2 & 0 & 0 & 0 & 0 & 0 & 0 & 0 & 0 \\
\hline$\Omega_{2}$ & 6 & 6 & 0 & 0 & -2 & -2 & 2 & 2 & 2 & 0 & 0 & -2 & 0 & 0 & 0 & 0 & 0 & 0 & 0 & 0 \\
\hline$\Omega_{3}$ & 6 & 6 & 0 & 0 & -2 & 2 & 0 & 0 & -2 & -2 & -2 & 0 & 2 & 0 & 0 & 0 & 0 & 0 & 0 & 0 \\
\hline$\Omega_{4}$ & 6 & 6 & 0 & 0 & -2 & 2 & 0 & 0 & -2 & 2 & 2 & 0 & -2 & 0 & 0 & 0 & 0 & 0 & 0 & 0 \\
\hline$\Pi_{1}$ & 8 & -8 & -4 & 4 & 0 & 0 & 0 & 0 & 0 & 0 & 0 & 0 & 0 & 0 & 1 & -1 & 1 & -1 & 0 & 0 \\
\hline$\Pi_{2}$ & 8 & -8 & 4 & -4 & 0 & 0 & 0 & 0 & 0 & 0 & 0 & 0 & 0 & 0 & 1 & -1 & -1 & 1 & 0 & 0 \\
\hline
\end{tabular}

Table II. Character Table of the Optimal Group $\mathcal{G}$ of the $4 \times 4$ model.

As the notation suggests, the irreps $A_{1}$ and $\tilde{A}_{1}$ both reduce to $A_{1}$, in $C_{4 v}$, while $B_{2}$ and $\tilde{B}_{2}$ both reduce to $B_{2}$. Table III shows how the irreps of $\mathcal{G}$ split in $C_{4 v}$.

\begin{tabular}{|c|c|}
\hline $\mathcal{G}$ & $C_{4 v}$ \\
\hline$A_{1}$ & $A_{1}$ \\
\hline$\tilde{A}_{1}$ & $A_{1}$ \\
\hline$B_{2}$ & $B_{2}$ \\
\hline$\tilde{B}_{2}$ & $B_{2}$ \\
\hline$\Gamma_{1}$ & $2 B_{2}$ \\
\hline$\Gamma_{2}$ & $2 A_{1}$ \\
\hline$\Sigma_{1}$ & $A_{1}+2 B_{1}$ \\
\hline$\Sigma_{2}$ & $A_{1}+2 B_{1}$ \\
\hline$\Sigma_{3}$ & $2 A_{2}+B_{2}$ \\
\hline$\Sigma_{4}$ & $2 A_{2}+B_{2}$ \\
\hline$\Lambda_{1}$ & $A_{1}+B_{1}+E$ \\
\hline$\Lambda_{2}$ & $A_{1}+B_{1}+E$ \\
\hline$\Lambda_{3}$ & $A_{2}+B_{2}+E$ \\
\hline$\Lambda_{4}$ & $A_{2}+B_{2}+E$ \\
\hline$\Omega_{1}$ & $A_{2}+B_{1}+2 E$ \\
\hline$\Omega_{2}$ & $A_{2}+B_{1}+2 E$ \\
\hline$\Omega_{3}$ & $A_{1}+B_{2}+2 E$ \\
\hline$\Omega_{4}$ & $A_{1}+B_{2}+2 E$ \\
\hline$\Pi_{1}$ & $2 A_{1}+2 B_{1}+2 E$ \\
\hline$\Pi_{2}$ & $2 A_{2}+2 B_{2}+2 E$ \\
\hline
\end{tabular}

Table III. Reduction of the irreps of Optimal Group $\mathcal{G}$ of the $4 \times 4$ model in the point Group. 
We call $\mathcal{G}$ the Optimal Group because it enables us to explain the degeneracy of the one-particle energy spectrum; in other terms, no accidental degeneracy of orbitals occours using $\mathcal{G}$. In the Table IV below we report the one-body eigenvalues for $t=-1$, the degeneracy and the symmetry of each eigenvector. Below, we shall find that $\mathcal{G}$ is also adequate to classify the many-body ground states. For pairs, the $W=0$ Theorem ensures that no double occupancy is possible in the irreps $\tilde{A}_{1}, B_{2}, \Gamma_{1}, \Gamma_{2}, \Sigma_{1}, \Sigma_{2}, \Sigma_{3}, \Sigma_{4}, \Lambda_{2}, \Lambda_{3}, \Omega_{1}, \Omega_{2}, \Omega_{3}, \Pi_{1}$ and $\Pi_{2}$.

\begin{tabular}{|c|c|c|}
\hline Energy & Irrep of $\mathcal{G}$ & Degeneracy \\
\hline 4 & $\tilde{B}_{2}$ & 1 \\
\hline 2 & $\Lambda_{4}$ & 4 \\
\hline 0 & $\Omega_{4}$ & 6 \\
\hline-2 & $\Lambda_{1}$ & 4 \\
\hline-4 & $A_{1}$ & 1 \\
\hline
\end{tabular}

Table IV. One-body spectrum for $t=-1$.

\section{IV. $L O C A L$ FORMALISM AT HALF FILLING}

As observed in the previous Sections, $W=0$ pairs predict the possible ground state symmetries of systems which differ from closed shells by a pair. Let us now consider how the above analysis extends to the doped half filled system. Let $\mathcal{S}_{h f}$ denote the set (or shell) of the $k$ wave vectors such that $\epsilon(k)=0$. At half filling $\left(N^{2}\right.$ holes) for $U=0$ the ground state has the $\mathcal{S}_{h f}$ shell half occupied, while all $|k\rangle$ orbitals such that $\epsilon(k)<0$ are filled. The $k$ vectors of $\mathcal{S}_{h f}$ lie on the square having vertices $( \pm \pi, 0)$ and $(0, \pm \pi)$; one readily realizes that the dimension of the set $\mathcal{S}_{h f}$, is $\left|\mathcal{S}_{h f}\right|=2 N-2$.

For $N=4$, the 6 wave vectors are $k_{1}=(\pi, 0), k_{2}=(0, \pi), k_{3}=(\pi / 2, \pi / 2), k_{4}=(\pi / 2,-\pi / 2), k_{5}=$ $(-\pi / 2,-\pi / 2)$ and $k_{6}=(-\pi / 2, \pi / 2)$.

$(0, \pi)$

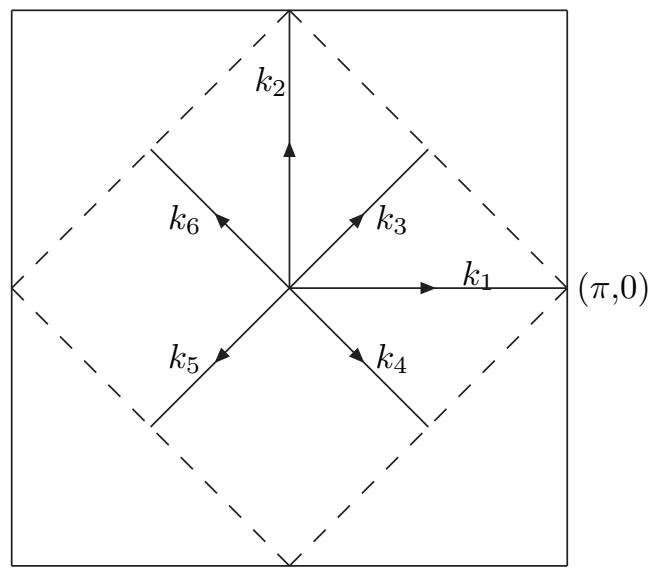

Figure I. The Brillouin Zone; the dashed square marks the condition of vanishing kinetic energy. $\mathcal{S}_{h f}$ contains 6 states (arrows) belonging to one-dimensional irreps of $\mathbf{T}$. Moreover, the arrows are mixed by operations of $C_{4 v}$, and $(\pi, 0)$ and $(0, \pi)$ are the basis of a two-dimensional irrep of $\mathbf{G}$, while the wavevectors $k=( \pm \pi / 2, \pm \pi / 2)$ mix among themselves and yield a four-dimensional irrep. The degeneracy of these two irreps is accidental for $\mathbf{G}$, but is explained by the Optimal Group $\mathcal{G}$. 
Since $H$ commutes with the total spin operators,

$$
\hat{S}_{z}=\frac{1}{2} \sum_{r}\left(\hat{n}_{r \uparrow}-\hat{n}_{r \downarrow}\right), \quad \hat{S}^{+}=\sum_{r} c_{r \uparrow}^{\dagger} c_{r \downarrow}, \quad \hat{S}^{-}=\left(\hat{S}^{+}\right)^{\dagger},
$$

at half filling every ground state of $H_{0}$ is represented in the $S_{z}=0$ subspace. Thus, $H_{0}$ has $\left(\begin{array}{l}6 \\ 3\end{array}\right)^{2}$ degenerate unperturbed ground state configurations with $S_{z}=0$. We will show how this degeneracy is removed by the Coulomb interaction $W$ already in first-order perturbation theory. Actually most of the degeneracy is removed in first-order, and with the help of Lieb's theorem [18] we shall be able to single out the true, unique first order ground state of $H$. In Appendix A we show that the structure of the first-order wave functions is gained by diagonalizing $W$ in the truncated Hilbert space $\mathcal{H}$ spanned by the states of 3 holes of each spin in $\mathcal{S}_{h f}$. In other terms, one solves a 6-particle problem in the truncated Hilbert space $\mathcal{H}$ and then, understanding the particles in the filled shells, obtains the first-order ground state eigenfunction of $H$ in the full 16-particle problem. We underline that the matrix of $H_{0}$ in $\mathcal{H}$ is null, since by construction $\mathcal{H}$ is contained in the kernel of $H_{0}$.

The operator $\sum_{r} \hat{n}_{r \uparrow} \hat{n}_{r \downarrow}$ has eigenvalues $0,1,2, \ldots$ and so the lowest eigenvalue of $W$ is zero (in other terms, $W$ is positive semi-definite). The unique ground state of the Hubbard Hamiltonian for $U=0^{+}$at half filling will turn out to be a $W=0$ singlet state of 6 holes in $\mathcal{S}_{h f}$ (filled shells being understood). We shall obtain the $W=0$ states $\in \mathcal{H}$. It is clear that, although the $U=0$ case is trivial, at $U=0^{+}$we are still facing a bona fide many-body problem, that we are solving exactly [20]. In the present Section we define a basis of local orbitals for the $4 \times 4$ Hubbard model with periodic boundary conditions; this basis is crucial for making the problem tractable, both at half filling and for the doped case. Using the local basis, the many body wave function of the antiferromagnetic ground state can be projected out as the singlet component of a single determinant, which is amazingly simple for an interacting system; the effective interaction between the doped holes also emerges analytically. The treatment for the half-filled case has already been generalized [21] to the $N \times N$ Hubbard model; below, we present the much simpler solution of the $4 \times 4$ case, which is sufficient for our present purposes.

Since $W$ depends on the occupation number operators $\hat{n}_{r}$, it is intuitive that its properties in $\mathcal{H}$ are best discussed by a suitable one-body basis of $\mathcal{S}_{h f}$ such that at least one of these operators is diagonal. In addition, a convenient basis should exploit the large $\mathbf{G}$ symmetry of the system. If $\mathcal{S}_{h f}$ were a complete set $\left(N^{2}=16\right.$ states), one would trivially go from plane waves to atomic orbitals by a Fourier transformation; instead, we must define the local counterparts of plane-wave states using only the $2 N-2=6$ states that belong to $\mathcal{S}_{h f}$.

For each site $r$ we diagonalize the number operator $\hat{n}_{r}$ (for the moment we omit the spin index); it is a trivial matter to verify that $\left(n_{r}\right)_{i j}=\left\langle k_{i}\left|\hat{n}_{r}\right| k_{j}\right\rangle=\frac{1}{16} e^{i\left(k_{i}-k_{j}\right) r}$ has eigenvalues $3 / 8$ and five times 0 . This degeneracy suggests that we should diagonalize other operators in order to label the $\hat{n}_{r}$ eigenvactors, and indeed, since $\hat{n}_{r}$ is compatible with the operations of the point symmetry group $C_{4 v}$ we also diagonalize the Dirac characters of this Group. The set of Dirac characters defines the irreducible representation (irrep); thus we write the one-body basis states $\left\{\left|\varphi_{\alpha}^{(r)}\right\rangle\right\}$ where $\alpha$ comprises the $\hat{n}_{r}$ eigenvalue and an $C_{4 v}$ irrep label. It is easy to verify that for $r=0$ the eigenvector with nonzero eigenvalue is just the totally symmetric superposition of all the $\left\{\left|k_{i}\right\rangle\right\} \in \mathcal{S}_{h f}$. Translating by $r$, plane wave states pick up a phase factor: $|k\rangle \rightarrow e^{i k r}|k\rangle$. Hence the $\hat{n}_{r}$ eigenvector of occupation $3 / 8$ is

$$
\left|\phi_{1}^{(r)}\right\rangle \equiv\left|\varphi_{A_{1}}^{(r)}\right\rangle=\frac{1}{\sqrt{6}} \sum_{j=1}^{6} e^{i k_{j} r}\left|k_{j}\right\rangle
$$

and we set up our local basis at $r$ by

$$
\begin{gathered}
\left|\phi_{i}^{(r)}\right\rangle=\sum_{j=1}^{6} O_{i j} e^{i k_{j} r}\left|k_{j}\right\rangle \\
\left|k_{j}\right\rangle=e^{-i k_{j} r} \sum_{n=1}^{6} O_{n j}\left|\phi_{n}^{(r)}\right\rangle
\end{gathered}
$$


where we introduce the orthogonal matrix

$$
O=\frac{1}{\sqrt{6}}\left[\begin{array}{rrrrrr}
1 & 1 & 1 & 1 & 1 & 1 \\
\sqrt{2} & \sqrt{2} & \frac{-1}{\sqrt{2}} & \frac{-1}{\sqrt{2}} & \frac{-1}{\sqrt{2}} & \frac{-1}{\sqrt{2}} \\
0 & 0 & \sqrt{\frac{3}{2}} & -\sqrt{\frac{3}{2}} & \sqrt{\frac{3}{2}} & -\sqrt{\frac{3}{2}} \\
\sqrt{3} & -\sqrt{3} & 0 & 0 & 0 & 0 \\
0 & 0 & \sqrt{3} & 0 & -\sqrt{3} & 0 \\
0 & 0 & 0 & \sqrt{3} & 0 & -\sqrt{3}
\end{array}\right]
$$

It is clear from Eqs.(23)(25) that $\left|\phi_{i}^{(r)}\right\rangle$ has well defined occupation $n$ and symmetry for point group operations centered at site $r$; namely, it has $n=3 / 8$ for $i=1$ and $n=0$ otherwise; it belongs to $A_{1}$ for $i=1,2$, to $B_{2}(x y)$ for $i=3$, to $B_{1}\left(x^{2}-y^{2}\right)$ for $i=4$ and to $E$ for $i=5,6$. The local bases of different sites $r$ and $r /$ are connected by the unitary transformation

$$
\left|\phi_{i}^{(r)}\right\rangle=\sum_{j=1}^{6}\left|\phi_{j}^{\left(r^{\prime}\right)}\right\rangle T_{j, i}^{\left(r^{\prime}, r\right)}
$$

and using the orthonormality of the $|k\rangle$ states we obtain the elements of the symmetric translation matrix

$$
T_{j, i}^{\left(r^{\prime}, r\right)}=\left\langle\phi_{j}^{\left(r^{\prime}\right)} \mid \phi_{i}^{(r)}\right\rangle=\sum_{m=1}^{6} O_{j, m} O_{i, m} e^{i k_{m} \cdot\left(r-r^{\prime}\right)}
$$

The translation matrix knows all the $\mathbf{G}$ symmetry of the system, and must be very special. Using such a basis set for the half filled shell the antiferromagnetic order of the ground state comes out in a clear and transparent manner. It is clear that $\left[T^{\left(r^{\prime}, r\right)}\right]^{4}=1$. The translation by one step towards the right is accomplished by

$$
T^{(\text {right })}=\left[\begin{array}{rrrrrr}
0 & 0 & 0 & \frac{-1}{\sqrt{3}} & \frac{i}{\sqrt{3}} & \frac{i}{\sqrt{3}} \\
0 & 0 & 0 & -\sqrt{\frac{2}{3}} & \frac{-i}{\sqrt{6}} & \frac{-i}{\sqrt{6}} \\
0 & 0 & 0 & 0 & \frac{i}{\sqrt{2}} & \frac{-i}{\sqrt{2}} \\
\frac{-1}{\sqrt{3}} & -\sqrt{\frac{2}{3}} & 0 & 0 & 0 & 0 \\
\frac{i}{\sqrt{3}} & \frac{-i}{\sqrt{6}} & \frac{i}{\sqrt{2}} & 0 & 0 & 0 \\
\frac{i}{\sqrt{3}} & \frac{-i}{\sqrt{6}} & \frac{-i}{\sqrt{2}} & 0 & 0 & 0
\end{array}\right]
$$

The matrix that makes one step upwards is

$$
T^{(u p)}=\left[\begin{array}{rrrrrr}
0 & 0 & 0 & \frac{1}{\sqrt{3}} & \frac{i}{\sqrt{3}} & \frac{-i}{\sqrt{3}} \\
0 & 0 & 0 & \sqrt{\frac{2}{3}} & \frac{-i}{\sqrt{6}} & \frac{i}{\sqrt{6}} \\
0 & 0 & 0 & 0 & \frac{i}{\sqrt{2}} & \frac{i}{\sqrt{2}} \\
\frac{1}{\sqrt{3}} & \sqrt{\frac{2}{3}} & 0 & 0 & 0 & 0 \\
\frac{i}{\sqrt{3}} & \frac{-i}{\sqrt{6}} & \frac{i}{\sqrt{2}} & 0 & 0 & 0 \\
\frac{-i}{\sqrt{3}} & \frac{i}{\sqrt{6}} & \frac{i}{\sqrt{2}} & 0 & 0 & 0
\end{array}\right]
$$

The reason why this choice of the basis set is clever is now apparent. The local basis at any site $r$ splits into the subsets $\mathcal{S}_{a}=\left\{\left|\phi_{1}^{(r)}\right\rangle,\left|\phi_{2}^{(r)}\right\rangle,\left|\phi_{3}^{(r)}\right\rangle\right\}$, and $\mathcal{S}_{b}=\left\{\left|\phi_{4}^{(r)}\right\rangle,\left|\phi_{5}^{(r)}\right\rangle,\left|\phi_{6}^{(r)}\right\rangle\right\}$; a shift by a lattice step sends members of $\mathcal{S}_{a}$ into linear combinations of the menbers of $\mathcal{S}_{b}$, and conversely.

Consider the 6-body determinantal eigenstate of $H_{0}$

$$
\left|d^{(r)}[1,2,3]\right\rangle_{\sigma}=\left|\phi_{1, \sigma}^{(r)} \phi_{2, \sigma}^{(r)} \phi_{3, \sigma}^{(r)} \phi_{4,-\sigma}^{(r)} \phi_{5,-\sigma}^{(r)} \phi_{6,-\sigma}^{(r)}\right\rangle ;
$$

the notation implies that $\left|d^{(r)}[i, j, k]\right\rangle_{\sigma}$ denotes a 6 -body determinant with one body per local state and $i, j, k$ with spin $\sigma$, the complement with spin $-\sigma$; local states are ordered in the natural way 
$1, \ldots 6$. In this state there is partial occupation of site $r$ with spin $\sigma$, but no double occupation. Introducing the primitive translation of the lattice $\hat{e}_{x}=(1,0)$ and $\hat{e}_{y}=(0,1)$, it turns out that a shift by a lattice step $r \rightarrow r^{\prime}=r \pm \hat{e}_{l}$ with $l=x, y$, produces the transformation

$$
\left|d^{(r)}[1,2,3]\right\rangle_{\sigma} \longleftrightarrow\left|d^{\left(r^{\prime}\right)}[1,2,3]\right\rangle_{\sigma}=-\left|d^{(r)}[4,5,6]\right\rangle_{\sigma},
$$

that is, a lattice step is equivalent to a spin flip (antiferromagnetic property). Since the spin-flipped state is also free of double occupation, $\left|d^{(r)}[1,2,3]\right\rangle_{\sigma}$ is a $W=0$-body eigenstate of $H$. A ground state which is a single determinant is a quite remarkable property of an interacting model like this, and this property holds at half filling, not in general. To be sure, $\left|d^{(r)}[1,2,3]\right\rangle_{\sigma}$ is a mixture of pure spin components $\left|\Phi_{A F}^{S}\right\rangle$ with $S=0,1,2,3$. However, $W$ is positive semi-definite and this implies that all the pure spin components must possess the $W=0$ property as well. In particular, the singlet $\left|\Phi_{A F}^{S=0}\right\rangle$ is a $W=0$ eigenstate and is the true ground state of the Hubbard model at half filling which is predicted by Lieb's theorem (filled shell are understood). Explicitly, the antiferromagnetic ground state wave function reads

$$
\left|\Phi_{A F}^{S=0}\right\rangle=\hat{A}(3,6) \hat{A}(2,5) \hat{A}(1,4)\left|d^{(r)}[1,2,3]\right\rangle_{\sigma},
$$

where $\hat{A}$ is the antisimmetrizer, such that for example $\hat{A}(1,4)\left|d^{(r)}[1,2,3]\right\rangle_{\sigma}=\left|d^{(r)}[1,2,3]\right\rangle_{\sigma}-$ $\left|d^{(r)}[4,2,3]\right\rangle_{\sigma}$. One can easly verify that $\left|\Phi_{A F}^{S=0}\right\rangle$ is independent by the $r$ and $\sigma$ label of $\left|d^{(r)}[1,2,3]\right\rangle_{\sigma}$, modulo phase factors. From the general analysis of Ref. [21] we obtain that this singlet has $A_{1}$ symmetry with respect to the center of an arbitrary plaquette of the square lattice and vanishing total momentum as in the strong coupling limit [22]. It is worth noticing that the open-shell part of the antiferromagnetic ground state (not considering the occupied inner shells) is a 6-body $W=0$ singlet state. Correlation effects enable no fewer than 6 particles to completely avoid double occupation in such a small system. This is also a consequence of Lieb's theorem. If all the 6 body are taken with parallel spin, double occupation is trivially avoided; however, Lieb's theorem enforces a singlet ground state, so a singlet $W=0$ state must exist.

The $4 \times 4$ case at hand can be thorougly explored on the computer, since the size of $\mathcal{H}$ at half filling is 400 . We have used Mathematica to diagonalize $H+\xi S^{2}$, where a small $\xi$ is a numerical device to keep the different spin components of the ground state separated. In this way, we observed the fourfold degenerate, $W=0$ ground state which $\xi$ separates into its singlet, triplet, quinted and septet components, as expected. At $\xi=0$ the separation grows like $U^{2}$. The antiferromagnetic property of the wave functions was also easily and nicely borne out by the numerical results.

\section{V. $W=0$ PAIRS AND QUARTETS IN THE PLANE-WAVE REPRESENTATION.}

In this Section we use the antiferromagnetic ground state $\left|\Phi_{A F}^{S=0}\right\rangle$ to predict the possible symmetries of the doped half filled system. With 12 holes, in the $U \rightarrow 0$ limit, there are two in $\mathcal{S}_{h f}$; the first-order ground states correspond to $W=0$ pairs. The symmetry of these $W=0$ states can be determined $a$ priori from the $\mathcal{G}$ irreps of Table IV. Apart from the filled shells, two holes go to the $\Omega_{4}$ level (Table IV). From the character Table II one can derive that

$$
\Omega_{4}^{2}=A_{1}+\tilde{B}_{2}+\Omega_{4}+\Gamma_{1}+\Gamma_{2}+\Sigma_{2}+\Sigma_{3}+\Omega_{1}+\Omega_{2}+\Omega_{3} ;
$$

since the first 3 entries are present in Table IV, the $W=0$ Theorem ensures that $\Gamma_{1}, \Gamma_{2}, \Sigma_{2}, \Sigma_{3}, \Omega_{1}, \Omega_{2}$ and $\Omega_{3}$ pairs have no double occupation. It turns out that the spin and orbital symmetries are entangled, i.e. some of these pairs are triplet and the rest singlet. We can see that by projecting the determinantal state $c_{k \uparrow}^{\dagger} c_{p \downarrow}^{\dagger}|0\rangle$ with $k, p \in \mathcal{S}_{h f}$ on the irreps not contained in the spectrum. One obtaines singlet $W=0$ pairs for $\Gamma_{1}, \Gamma_{2}, \Sigma_{2}, \Omega_{1}$; they read

$$
\begin{aligned}
& \left|\psi_{1}^{1} \Gamma_{1}\right\rangle=\left\{\frac{1}{\sqrt{3}}\left(c_{k_{1} \uparrow}^{\dagger} c_{k_{2} \downarrow}^{\dagger}+c_{k_{2} \uparrow}^{\dagger} c_{k_{1} \downarrow}^{\dagger}\right)-\frac{1}{2 \sqrt{3}}\left(c_{k_{3} \uparrow}^{\dagger} c_{k_{3} \downarrow}^{\dagger}-c_{k_{4} \uparrow}^{\dagger} c_{k_{4} \downarrow}^{\dagger}+c_{k_{5} \uparrow}^{\dagger} c_{k_{5} \downarrow}^{\dagger}-c_{k_{6} \uparrow}^{\dagger} c_{k_{6} \downarrow}^{\dagger}\right)\right\}|0\rangle \\
& \left|\psi_{2}^{1} \Gamma_{1}\right\rangle=\frac{1}{2}\left(c_{k_{3} \uparrow}^{\dagger} c_{k_{5} \downarrow}^{\dagger}-c_{k_{4} \uparrow}^{\dagger} c_{k_{6} \downarrow}^{\dagger}+c_{k_{5} \uparrow}^{\dagger} c_{k_{3} \downarrow}^{\dagger}-c_{k_{6} \uparrow}^{\dagger} c_{k_{4} \downarrow}^{\dagger}\right)|0\rangle \\
& \left|\psi_{1}^{1} \Gamma_{2}\right\rangle=\left\{\frac{1}{\sqrt{3}}\left(c_{k_{1} \uparrow}^{\dagger} c_{k_{1} \downarrow}^{\dagger}+c_{k_{2} \uparrow}^{\dagger} c_{k_{2} \downarrow}^{\dagger}\right)+\frac{1}{2 \sqrt{3}}\left(c_{k_{3} \uparrow}^{\dagger} c_{k_{5} \downarrow}^{\dagger}+c_{k_{4} \uparrow}^{\dagger} c_{k_{6} \downarrow}^{\dagger}+c_{k_{5} \uparrow}^{\dagger} c_{k_{3} \downarrow}^{\dagger}+c_{k_{6} \uparrow}^{\dagger} c_{k_{4} \downarrow}^{\dagger}\right)\right\}|0\rangle \\
& \left|\psi_{2}^{1} \Gamma_{2}\right\rangle=\frac{1}{2}\left(c_{k_{3} \uparrow}^{\dagger} c_{k_{3} \downarrow}^{\dagger}+c_{k_{4} \uparrow}^{\dagger} c_{k_{4} \downarrow}^{\dagger}+c_{k_{5} \uparrow}^{\dagger} c_{k_{5} \downarrow}^{\dagger}+c_{k_{6} \uparrow}^{\dagger} c_{k_{6} \downarrow}^{\dagger}\right)|0\rangle
\end{aligned}
$$




$$
\begin{aligned}
\left|\psi_{1}^{1} \Sigma_{2}\right\rangle & =\frac{1}{\sqrt{2}}\left(c_{k_{1} \uparrow}^{\dagger} c_{k_{1} \downarrow}^{\dagger}-c_{k_{2} \uparrow}^{\dagger} c_{k_{2} \downarrow}^{\dagger}\right)|0\rangle \\
\left|\psi_{2}^{1} \Sigma_{2}\right\rangle & =\frac{1}{2}\left(c_{k_{3} \uparrow}^{\dagger} c_{k_{4} \downarrow}^{\dagger}+c_{k_{4} \uparrow}^{\dagger} c_{k_{3} \downarrow}^{\dagger}+c_{k_{5} \uparrow}^{\dagger} c_{k_{6} \downarrow}^{\dagger}+c_{k_{6} \uparrow}^{\dagger} c_{k_{5} \downarrow}^{\dagger}\right)|0\rangle \\
\left|\psi_{3}^{1} \Sigma_{2}\right\rangle & =\frac{1}{2}\left(c_{k_{3} \uparrow}^{\dagger} c_{k_{6} \downarrow}^{\dagger}+c_{k_{4} \uparrow}^{\dagger} c_{k_{5} \downarrow}^{\dagger}+c_{k_{5} \uparrow}^{\dagger} c_{k_{4} \downarrow}^{\dagger}+c_{k_{6} \uparrow}^{\dagger} c_{k_{3} \downarrow}^{\dagger}\right)|0\rangle
\end{aligned}
$$

and finally

$$
\begin{aligned}
& \left|\psi_{1}^{1} \Omega_{1}\right\rangle=\frac{1}{2}\left(c_{k_{1} \uparrow}^{\dagger} c_{k_{3} \downarrow}^{\dagger}+c_{k_{3} \uparrow}^{\dagger} c_{k_{1} \downarrow}^{\dagger}+c_{k_{2}}^{\dagger} c_{k_{5} \downarrow}^{\dagger}+c_{k_{5} \uparrow}^{\dagger} c_{k_{2} \downarrow}^{\dagger}\right)|0\rangle \\
& \left|\psi_{2}^{1} \Omega_{1}\right\rangle=\frac{1}{2}\left(c_{k_{1} \uparrow}^{\dagger} c_{k_{4} \downarrow}^{\dagger}+c_{k_{4} \uparrow}^{\dagger} c_{k_{1} \downarrow}^{\dagger}-c_{k_{2} \uparrow}^{\dagger} k_{k_{6} \downarrow}^{\dagger}-c_{k_{6} \uparrow}^{\dagger} c_{k_{2} \downarrow}^{\dagger}\right)|0\rangle \\
& \left|\psi_{3}^{1} \Omega_{1}\right\rangle=\frac{1}{2}\left(c_{k_{1} \uparrow}^{\dagger} c_{k_{5} \downarrow}^{\dagger}+c_{k_{5} \uparrow}^{\dagger} c_{k_{1} \downarrow}^{\dagger}+c_{k_{2} \uparrow}^{\dagger} c_{k_{3} \downarrow}^{\dagger}+c_{k_{3} \uparrow}^{\dagger} c_{k_{2} \downarrow}^{\dagger}\right)|0\rangle \\
& \left|\psi_{4}^{1} \Omega_{1}\right\rangle=\frac{1}{2}\left(c_{k_{1} \uparrow}^{\dagger} c_{k_{6} \downarrow}^{\dagger}+c_{k_{6} \uparrow}^{\dagger} c_{k_{1} \downarrow}^{\dagger}-c_{k_{2} \uparrow}^{\dagger} c_{k_{4} \downarrow}^{\dagger}-c_{k_{4} \uparrow}^{\dagger} c_{k_{2} \downarrow}^{\dagger}\right)|0\rangle \\
& \left|\psi_{5}^{{ }_{5} \Omega_{1}}\right\rangle=\frac{1}{\sqrt{2}}\left(c_{k_{3} \uparrow}^{\dagger} c_{k_{3} \downarrow}^{\dagger}-c_{k_{5} \uparrow}^{\dagger} c_{k_{5} \downarrow}^{\dagger}\right)|0\rangle \\
& \left|\psi_{6}^{1} \Omega_{1}\right\rangle=\frac{1}{\sqrt{2}}\left(c_{k_{4} \uparrow}^{\dagger} c_{k_{4} \downarrow}^{\dagger}-c_{k_{6} \uparrow}^{\dagger} c_{k_{6} \downarrow}^{\dagger}\right)|0\rangle .
\end{aligned}
$$

Other irreps yield $W=0$ triplet pairs. They are the three times degenerate irrep

$$
\begin{aligned}
& \left|\psi_{1}^{3} \Sigma_{3}\right\rangle=\frac{1}{\sqrt{2}}\left(c_{k_{1} \uparrow}^{\dagger} c_{k_{2} \downarrow}^{\dagger}-c_{k_{2} \uparrow}^{\dagger} c_{k_{1} \downarrow}^{\dagger}\right)|0\rangle \\
& \left|\psi_{2}^{{ }^{3} \Sigma_{3}}\right\rangle=\frac{1}{2}\left(c_{k_{3} \uparrow}^{\dagger} c_{k_{4} \downarrow}^{\dagger}-c_{k_{4} \uparrow}^{\dagger} c_{k_{3} \downarrow}^{\dagger}+c_{k_{5} \uparrow}^{\dagger} c_{k_{6} \downarrow}^{\dagger}-c_{k_{6} \uparrow}^{\dagger} c_{k_{5} \downarrow}^{\dagger}\right)|0\rangle \\
& \left|\psi_{3}{ }^{3} \Sigma_{3}\right\rangle=\frac{1}{2}\left(c_{k_{3} \uparrow}^{\dagger} c_{k_{6} \downarrow}^{\dagger}-c_{k_{4} \uparrow}^{\dagger} c_{k_{5} \downarrow}^{\dagger}+c_{k_{5} \uparrow}^{\dagger} c_{k_{4} \downarrow}^{\dagger}-c_{k_{6} \uparrow}^{\dagger} c_{k_{3} \downarrow}^{\dagger}\right)|0\rangle
\end{aligned}
$$

and the two sixfold sets

$$
\begin{aligned}
& \left|\psi_{1}^{3} \Omega_{2}\right\rangle=\frac{1}{2}\left(c_{k_{1} \uparrow}^{\dagger} c_{k_{3} \downarrow}^{\dagger}-c_{k_{3} \uparrow}^{\dagger} c_{k_{1} \downarrow}^{\dagger}+c_{k_{2} \uparrow}^{\dagger} c_{k_{5} \downarrow}^{\dagger}-c_{k_{5} \uparrow}^{\dagger} c_{k_{2} \downarrow}^{\dagger}\right)|0\rangle \\
& \left|\psi_{2}^{3} \Omega_{2}\right\rangle=\frac{1}{2}\left(c_{k_{1} \uparrow}^{\dagger} c_{k_{4} \downarrow}^{\dagger}-c_{k_{4} \uparrow}^{\dagger} c_{k_{1} \downarrow}^{\dagger}-c_{k_{2} \uparrow}^{\dagger} c_{k_{6} \downarrow}^{\dagger}+c_{k_{6}}^{\dagger} c_{k_{2} \downarrow}^{\dagger}\right)|0\rangle \\
& \left|\psi_{3}^{3} \Omega_{2}\right\rangle=\frac{1}{2}\left(c_{k_{1} \uparrow}^{\dagger} c_{k_{5} \downarrow}^{\dagger}-c_{k_{5} \uparrow}^{\dagger} c_{k_{1} \downarrow}^{\dagger}+c_{k_{2} \uparrow}^{\dagger} c_{k_{3} \downarrow}^{\dagger}-c_{k_{3} \uparrow}^{\dagger} c_{k_{2} \downarrow}^{\dagger}\right)|0\rangle \\
& \left|\psi_{4}^{3} \Omega_{2}\right\rangle=\frac{1}{2}\left(c_{k_{1} \uparrow}^{\dagger} c_{k_{6} \downarrow}^{\dagger}-c_{k_{6} \uparrow}^{\dagger} c_{k_{1} \downarrow}^{\dagger}-c_{k_{2} \uparrow}^{\dagger} c_{k_{4} \downarrow}^{\dagger}+c_{k_{4} \uparrow}^{\dagger} c_{k_{2} \downarrow}^{\dagger}\right)|0\rangle \\
& \left|\psi_{5}^{3} \Omega_{2}\right\rangle=\frac{1}{\sqrt{2}}\left(c_{k_{3} \uparrow}^{\dagger} c_{k_{5} \downarrow}^{\dagger}-c_{k_{5} \uparrow}^{\dagger} c_{k_{3} \downarrow}^{\dagger}\right)|0\rangle \\
& \left|\psi_{6}^{3} \Omega_{2}\right\rangle=\frac{1}{\sqrt{2}}\left(c_{k_{4} \uparrow}^{\dagger} \uparrow c_{k_{6} \downarrow}^{\dagger}-c_{k_{6} \uparrow}^{\dagger} c_{k_{4} \downarrow}^{\dagger}\right)|0\rangle
\end{aligned}
$$

and

$$
\begin{aligned}
& \left|\psi_{1}^{3} \Omega_{3}\right\rangle=\frac{1}{2}\left(c_{k_{1} \uparrow}^{\dagger} c_{k_{3} \downarrow}^{\dagger}-c_{k_{3} \uparrow}^{\dagger} c_{k_{1} \downarrow}^{\dagger}-c_{k_{2} \uparrow}^{\dagger} c_{k_{5} \downarrow}^{\dagger}+c_{k_{5} \uparrow}^{\dagger} c_{k_{2} \downarrow}^{\dagger}\right)|0\rangle \\
& \left|\psi_{2}^{3} \Omega_{3}\right\rangle=\frac{1}{2}\left(c_{k_{1} \uparrow}^{\dagger} c_{k_{4} \downarrow}^{\dagger}-c_{k_{4} \uparrow}^{\dagger} c_{k_{1} \downarrow}^{\dagger}+c_{k_{2} \uparrow}^{\dagger} c_{k_{6} \downarrow}^{\dagger}-c_{k_{6} \uparrow}^{\dagger} c_{k_{2} \downarrow}^{\dagger}\right)|0\rangle \\
& \left|\psi_{3}^{3} \Omega_{3}\right\rangle=\frac{1}{2}\left(c_{k_{1} \uparrow}^{\dagger} c_{k_{5} \downarrow}^{\dagger}-c_{k_{5} \uparrow}^{\dagger} c_{k_{1} \downarrow}^{\dagger}-c_{k_{2} \uparrow}^{\dagger} c_{k_{3} \downarrow}^{\dagger}+c_{k_{3} \uparrow}^{\dagger} c_{k_{2} \downarrow}^{\dagger}\right)|0\rangle \\
& \left|\psi_{4}^{3} \Omega_{3}\right\rangle=\frac{1}{2}\left(c_{k_{1} \uparrow}^{\dagger} c_{k_{6} \downarrow}^{\dagger}-c_{k_{6} \uparrow}^{\dagger} c_{k_{1} \downarrow}^{\dagger}+c_{k_{2} \uparrow}^{\dagger} c_{k_{4} \downarrow}^{\dagger}-c_{k_{4} \uparrow}^{\dagger} c_{k_{2} \downarrow}^{\dagger}\right)|0\rangle \\
& \left|\psi_{5}^{3} \Omega_{3}\right\rangle=\frac{1}{2}\left(c_{k_{3} \uparrow}^{\dagger} c_{k_{4} \downarrow}^{\dagger}-c_{k_{4} \uparrow}^{\dagger} c_{k_{3} \downarrow}^{\dagger}+c_{k_{6} \uparrow}^{\dagger} c_{k_{5} \downarrow}^{\dagger}-c_{k_{5} \uparrow}^{\dagger} c_{k_{6} \downarrow}^{\dagger}\right)|0\rangle \\
& \left|\psi_{6}^{3} \Omega_{3}\right\rangle=\frac{1}{2}\left(c_{k_{3} \uparrow}^{\dagger} c_{k_{6} \downarrow}^{\dagger}-c_{k_{6} \uparrow}^{\dagger} c_{k_{3} \downarrow}^{\dagger}+c_{k_{4} \uparrow}^{\dagger} c_{k_{5} \downarrow}^{\dagger}-c_{k_{5} \uparrow}^{\dagger} c_{k_{4} \downarrow}^{\dagger}\right)|0\rangle .
\end{aligned}
$$

The above a priori argument hardly applies to the symmetries of $W=0$ quartets, because $\Omega_{4}^{4}$ contains almost every symmetry and we do not know any $W=0$-like Theorem for quartets. However, we can still build the projection operators by Mathematica; we can project the 225 quartets on the irreps of $\mathcal{G}$ and carry on the analysis in an efficient, if not elegant, way. We found that the singlet $W=0$ quartets are 13 as many as the singlet $W=0$ pairs, and belong to the same irreps $\Gamma_{1}, \Gamma_{2}, \Sigma_{2}, \Omega_{1}$. Therefore, these are the possible symmetries of the first-order ground states with 14 holes. Exact diagonalization results [2] show that for $U / t<3$ and $16-2=14$ holes the ground state is sixfold degenerate, with a doublet of states with momentum $(\pi, \pi)$ and a quartet with momentum $( \pm \pi / 2, \pm \pi / 2)$. In view of Eqs.(37), the computed ground state corresponds to an $\Omega_{1}$ electron pair over the half filled system. For $U / t>3$ and the same number of holes a level crossing takes place: the ground state is threefold degenerate and contains a state with momentum $(0,0)$ and a doublet with momentum $(\pi, 0)$ and $(0, \pi)$. In view of Eqs.(36), the computed ground state must be assigned to a $\Sigma_{2}$ electron pair over the half filled system. In both cases, the symmetry of the ground state corresponds to a $W=0$ pair. 


\section{LOCAL PICTURE OF THE $W=0$ PAIRS AND QUARTETS}

The antiferromagnetic property of the local basis of any site $r$ readily prompts $W=0$ pairs; alternatively, we can transform the pairs and quartets of well-defined symmetry (Section V) using the local basis. Both methods are useful. The vacuum at half filling is a $W=0$ state with 6 holes. The Quartets are then obtained from the antiferromagnetic ground state at half filling by removing a $W=0$ pair.

\section{A. $\mathbf{W}=0$ Pairs}

Chosen a site $r$, let $\left|\phi_{\alpha}^{(r)}\right\rangle$ be a normalized linear combination of the states $\left|\phi_{1}^{(r)}\right\rangle,\left|\phi_{2}^{(r)}\right\rangle,\left|\phi_{3}^{(r)}\right\rangle$ of $\mathcal{S}_{a}$ and $\phi_{\beta}^{(r)}$ be a normalized linear combination of $\left|\phi_{4}^{(r)}\right\rangle,\left|\phi_{5}^{(r)}\right\rangle,\left|\phi_{6}^{(r)}\right\rangle$ of $\mathcal{S}_{b}$. Then,

$$
\left|d_{\alpha, \beta}^{(r)}\right\rangle=\left|\phi_{\alpha \uparrow}^{(r)} \phi_{\beta \downarrow}^{(r)}\right\rangle
$$

is a two-body state free of double occupation on every site and $\frac{\left|\phi_{\alpha \uparrow}^{(r)} \phi_{\beta \downarrow}^{(r)}\right\rangle+\left|\phi_{\beta \uparrow}^{(r)} \phi_{\alpha \downarrow}^{(r)}\right\rangle}{\sqrt{2}}$ is a $W=0$ pair. Since for each spin one has 3 degrees of freedom, one can build 9 independent pairs in this way; they are bases for the $\Omega_{1}$ and $\Sigma_{2} W=0$ pairs of the previous Section. The only alternative method for obtaining $W=0$ pairs is that of forming $\left|\phi_{\alpha}^{(r)}\right\rangle$ and $\left|\phi_{\beta}^{(r)}\right\rangle$ as linear combinations of states of the same subset (both from $\mathcal{S}_{a}$ or both from $\mathcal{S}_{b}$ ). This can be accomplished in such a way that $\left|\phi_{1}^{(r)}\right\rangle$ never appears for both spins; actually, the bases of $\Gamma_{1}$ and $\Gamma_{2}$ are obtained in this way. If we use $\mathcal{S}_{a}$ for both spin directions then after a lattice step in any direction the pair is formed exclusively with states of $\mathcal{S}_{b}$ and its occupation vanishes; such pairs live on a sublattice.

We rewrite the pairs and quartets of Section V using the local basis of any site $r$. By Eq.(24),

$$
\left|k_{i} \uparrow k_{j} \downarrow\right\rangle=\sum_{m, n=1}^{6} e^{-i\left(k_{i}+k_{j}\right) r} O_{n i} O_{m j}\left|\phi_{n \uparrow}^{(r)} \phi_{m \downarrow}^{(r)}\right\rangle .
$$

For instance, the operator that annihilates the 6 -th component of the $\Omega_{1} W=0$ pair in Eq.(37) becomes

$$
\Psi_{6}^{1} \Omega_{1}=-\frac{1}{\sqrt{6}}\left(c_{1 \uparrow} c_{6 \downarrow}+c_{6 \uparrow} c_{1 \downarrow}\right)+\frac{1}{2}\left(c_{6 \uparrow} c_{3 \downarrow}+c_{3 \uparrow} c_{6 \downarrow}\right)+\frac{1}{2 \sqrt{3}}\left(c_{2 \uparrow} c_{6 \downarrow}+c_{6 \uparrow} c_{2 \downarrow}\right),
$$

where $c_{i \sigma}^{(r)^{\dagger}}$ and $c_{i \sigma}^{(r)}$ are hole creation and annihilation operators in the local states $\phi_{i \sigma}^{(r)}$; in Eq.(43) the site is not specified since whatever it is $\Psi_{6}^{1} \Omega_{1}$ does not change. The local representation of symmetryadapted pairs is of interest because different irreps are well characterized by their local behaviour.

\section{B. $\mathrm{W}=0$ Quartets}

With 14 holes, in the $U \rightarrow 0$ limit, there are four in $\mathcal{S}_{h f}$; the first-order ground states correspond to $W=0$ quartets. By removing in all possible ways two holes of opposite spin from the 6 -body $W=0$ determinant $\left|d^{(r)}[1,2,3]\right\rangle_{\sigma}$ of equation (30) one produces nine 4-body determinants. They are free of double occupation on site $r$ because $\left|\phi_{1}^{(r)}\right\rangle$ cannot appear for both spins; this property holds on all sites because of the special form of the translation matrices (28) and (29). It follows that these are $W=0$ states and in first-order perturbation theory they belong to the ground state multiplet. It is clear that these 9 first-order ground states are in one-to-one correspondence to the pairs (41), and for instance $\left|\phi_{2 \downarrow}^{(r)} \phi_{3 \downarrow}^{(r)} \phi_{5 \uparrow}^{(r)} \phi_{6 \uparrow}^{(r)}\right\rangle$ corresponds to the pair $\left|\phi_{1 \downarrow}^{(r)} \phi_{4 \uparrow}^{(r)}\right\rangle$; since $\left|\Phi_{A F}^{S=0}\right\rangle$ transforms as the totalsymmetric one-dimensional irrep $A_{1}$ under the $C_{4 v}$ operations referred to the center of a plaquette, the symmetries of the 9 quartets and the 9 pairs are also the same, namely, they are $\Omega_{1}$ and $\Sigma_{2}$ of $\mathcal{G}$. The total momentun labels are also the same. There is actually a complete correspondence between $W=0$ pairs and quartets; the quartets are also 13 and those that were not obtained above belong 
to $\Gamma_{1}$ and $\Gamma_{2}$. These cannot arise in the same way because one can show that they are not obtained by removing two holes from $\left|d^{(r)}[1,2,3]\right\rangle_{\sigma}$. This means that $\Gamma_{1}$ and $\Gamma_{2}$ are not to be interpreted as pairing states.

\section{PAIRING MECHANISM}

We consider the ground state of the $4 \times 4$ model with 14 holes; aside from the 10 holes in the inner $A_{1}$ and $\Lambda_{1}$ shells (see Table IV) the outer $\Omega_{4}$ shell contains 4 holes in a $W=0$ quartet. We are in position to show that the principles of Section IB produce a diagnosis of pairing between two electrons added to the antiferromagnetic 16 -holes ground state (half filling).

We recall from Section $V$ that, by comparing with exact diagonalization results [2], the ground state is assigned to $\Omega_{1}$, Eq.(37), at weak coupling and to $\Sigma_{2}$, Eq.(36), at a stronger coupling. In analogy with the points $a$ )-c) of Section IB, we must preliminarily verify that symmetry does not forbid obtaining these symmetries by creating $W=0$ electron pairs, Eqs.(34-40), from the antiferromagnetic state of Eq.(32). This is the same as annihilating hole pairs. Since the state $\left|\Phi_{A F}^{S=0}\right\rangle$ of Eq.(32) is a total symmetric singlet with vanishing total momentum, the labels of the quartets will be the same of the annihilated hole pairs. This operation can be done by hand, or with the help of Mathematica, and the answer to the preliminary question is adfirmative for $\Omega_{1}$ and $\Sigma_{2}$, but not for all pairs. We obtain $24 W=0$ quartets out of the 28 states in Eqs.(34-40), since the annihilation of $\Gamma_{1}$ and $\Gamma_{2}$ $W=0$ pairs gives identically zero.

We need to modify the canonical transformation to deal with the $4 \times 4$ cluster with periodic boundary conditions near half filling; the previous form is not adequate because the $\mathcal{S}_{h f}$ shell is only partially occupied and so in Eqs.(2,3) we have to use the antiferromagnetic state $\left|\Phi_{A F}^{S=0}\right\rangle$ of Eq.(32). In small clusters like the $4 \times 4$ one the one-body states are widely separated and the intra-shell interaction is much more important than the inter-shell one; therefore, we consider only the $m$ states made removing two holes in $\mathcal{S}_{h f}$ from $\left|\Phi_{A F}^{S=0}\right\rangle$, neglecting the high-lying unoccupied orbitals [17].

We now come to item $d$ ) of Section IB, the identification of the two states which are obtained by spin-flip from each other and are coupled by the interaction in second order. The explicit form of the symmetry adapted pairs of Section $\mathrm{V}$ shows that $\left|\psi_{1}^{1} \Sigma_{2}\right\rangle$ and $\left|\psi_{1}^{3} \Sigma_{3}\right\rangle$ involve only $k_{1}$ and $k_{2}$. This suggests that by taking linear combinations we can obtain a two-body state and its spin-flipped image. Specifically, using the electron creation operators

$$
\alpha_{\sigma}^{[ \pm]} \equiv \frac{1}{\sqrt{2}}\left(c_{k_{1} \sigma} \pm c_{k_{2} \sigma}\right)
$$

we build the following two-electron $W=0$ determinants:

$$
\left|w_{1}\right\rangle=\alpha_{\uparrow}^{[+]} \alpha_{\downarrow}^{[-]}|0\rangle_{e} \quad, \quad\left|w_{2}\right\rangle=\alpha_{\uparrow}^{[-]} \alpha_{\downarrow}^{[+]}|0\rangle_{e} .
$$

where the state $|0\rangle_{e}$ is the electron-vacuum state: $c_{k \sigma}^{\dagger}|0\rangle_{e}=0, \forall k, \sigma$. With Eq.(45) we can build the following $m$ states:

$$
\left|m_{1}\right\rangle=\alpha_{\uparrow}^{[+]} \alpha_{\downarrow}^{[-]}\left|\Phi_{A F}^{S=0}\right\rangle \quad, \quad\left|m_{2}\right\rangle=\alpha_{\uparrow}^{[-]} \alpha_{\downarrow}^{[+]}\left|\Phi_{A F}^{S=0}\right\rangle .
$$

Since $\left|m_{1}\right\rangle$ and $\left|m_{2}\right\rangle$ have projection only on irreps $\Sigma_{2}$ and $\Sigma_{3}$, the states (46) can be mixed by the operator $W_{\text {eff }}$ only between themselves, and so they are the only states involved in the canonical transformation. The eigenvalue equation (12) reduces to a $2 \times 2$ problem; the $W_{\text {eff }}$ matrix to diagonalize is:

$$
\left[\begin{array}{cc}
0 & \left\langle m_{1}\left|W_{\text {eff }}\right| m_{2}\right\rangle \\
\left\langle m_{2}\left|W_{\text {eff }}\right| m_{1}\right\rangle & 0
\end{array}\right] .
$$

The eigenvalues are $\pm\left\langle m_{1}\left|W_{e f f}\right| m_{2}\right\rangle$ and the eigenvectors are $\frac{1}{\sqrt{2}}(1, \pm 1)$. Expanding these eigenvectors in the base (46), we find:

$$
\left|\Psi_{1}^{1} \Sigma_{2}\right\rangle=\frac{1}{\sqrt{2}}\left(\left|m_{1}\right\rangle+\left|m_{2}\right\rangle\right) \equiv\left|\psi_{1}^{1} \Sigma_{2}\right\rangle_{e} \otimes\left|\Phi_{A F}^{S=0}\right\rangle, \quad\left|\Psi_{1}^{3} \Sigma_{3}\right\rangle=\frac{1}{\sqrt{2}}\left(\left|m_{1}\right\rangle-\left|m_{2}\right\rangle\right) \equiv\left|\psi_{1}^{3} \Sigma_{3}\right\rangle_{e} \otimes\left|\Phi_{A F}^{S=0}\right\rangle
$$


where the notation $\left|\psi_{i}^{\eta}\right\rangle_{e} \otimes\left|\Phi_{A F}^{S=0}\right\rangle$ stands for creating the electron-pair $\left|\psi_{i}^{\eta}\right\rangle$ over the antiferromagnetic state $\left|\Phi_{A F}^{S=0}\right\rangle$ (filled shells are understood) to get a 14 hole state. We use this example to stress that pairing by the present mechanism is possible only if the Optimal Group $\mathcal{G}$ possesses twin singlet and triplet representations with the same number of components, built by the same orbitals.

In a similar way, $\left|\psi_{6}^{1} \Omega_{1}\right\rangle$ and $\left|\psi_{6}^{3} \Omega_{2}\right\rangle$ involve only $k_{4}$ and $k_{6}$; this suggests introducing the electron creation operators

$$
\beta_{\sigma}^{[ \pm]} \equiv \frac{1}{\sqrt{2}}\left(c_{k_{4} \sigma} \pm c_{k_{6} \sigma}\right)
$$

the two-electron $W=0$ determinants

$$
\left|w_{1}^{\prime}\right\rangle=\beta_{\uparrow}^{[+]} \beta_{\downarrow}^{[-]}|0\rangle_{e} \quad, \quad\left|w_{2}^{\prime}\right\rangle=\beta_{\uparrow}^{[-]} \beta_{\downarrow}^{[+]}|0\rangle_{e}
$$

are obtained from each other by spin-flip. Incidentally, the latter property is preserved if one switches to the local picture by the transformation (24): introducing a new orbital

$$
c_{\Omega_{6} \sigma}=\frac{-c_{1 \sigma}}{\sqrt{3}}+\frac{c_{5 \sigma}}{\sqrt{2}}+\frac{c_{6 \sigma}}{\sqrt{6}}
$$

one finds

$$
\left|w_{1}^{\prime}\right\rangle=c_{\Omega_{6} \uparrow} c_{6 \downarrow}|0\rangle_{e},
$$

while

$$
\left|w_{2}^{\prime}\right\rangle=c_{6 \uparrow} c_{\Omega_{6} \downarrow}|0\rangle_{e}
$$

Thus, we have identified the $m$ states

$$
\left|m_{1}^{\prime}\right\rangle=\beta_{\uparrow}^{[+]} \beta_{\downarrow}^{[-]}\left|\Phi_{A F}^{S=0}\right\rangle \quad, \quad\left|m_{2}^{\prime}\right\rangle=\beta_{\uparrow}^{[-]} \beta_{\downarrow}^{[+]}\left|\Phi_{A F}^{S=0}\right\rangle .
$$

Since $\left|m_{1}^{\prime}\right\rangle$ and $\left|m_{2}^{\prime}\right\rangle$ have projection only on irreps $\Omega_{1}$ and $\Omega_{2}$, the states (54) can be mixed by the operator $W_{\text {eff }}$ only between themselves, and we find another $2 \times 2$ matrix

$$
\left[\begin{array}{cc}
0 & \left\langle m_{1}^{\prime}\left|W_{\text {eff }}\right| m_{2}^{\prime}\right\rangle \\
\left\langle m_{2}^{\prime}\left|W_{\text {eff }}\right| m_{1}^{\prime}\right\rangle & 0
\end{array}\right]
$$

with eigenvalues $\pm\left\langle m_{1}^{\prime}\left|W_{e f f}\right| m_{2}^{\prime}\right\rangle$ and eigenvectors $\frac{1}{\sqrt{2}}(1, \pm 1)$. Therefore,

$$
\left|\Psi_{2}^{1} \Omega_{1}\right\rangle=\frac{1}{\sqrt{2}}\left(\left|m_{1}^{\prime}\right\rangle+\left|m_{2}^{\prime}\right\rangle\right) \equiv\left|\psi_{6}^{1} \Omega_{1}\right\rangle_{e} \otimes\left|\Phi_{A F}^{S=0}\right\rangle, \quad\left|\Psi_{6}^{3} \Omega_{2}\right\rangle=\frac{1}{\sqrt{2}}\left(\left|m_{1}^{\prime}\right\rangle-\left|m_{2}^{\prime}\right\rangle\right) \equiv\left|\psi_{6}^{3} \Omega_{2}\right\rangle_{e} \otimes\left|\Phi_{A F}^{S=0}\right\rangle .
$$

The numerical values of the eigenvalues for $U=-t=1 \mathrm{eV}$ are negative in both cases,

$$
\left\langle m_{1}\left|W_{e f f}\right| m_{2}\right\rangle=-60.7 \mathrm{meV} \quad\left\langle m_{1}^{\prime}\left|W_{\text {eff }}\right| m_{2}^{\prime}\right\rangle=-61.9 \mathrm{meV}
$$

which means that in both the cases the Cooper-like equation (12) gives singlet ground states; the triplets have an opposite correction to the energy. The binding energy is larger for the $\left|\psi^{1} \Omega_{1}\right\rangle$ singlet, which is the true ground state (the result cannot depend on the component of the irrep). Moreover since $\left|\Phi_{A F}^{S=0}\right\rangle$ is a totalsymmetric singlet with vanishing total momentum, we can also predict momentum, spin and symmetry of the ground state. Our approach has enough predictive power to yield the symmetry, wavevector and degeneracy of the ground state.

\section{CONCLUSIONS}

Recently, we have proposed a new approach [15], [16] to the question of the existence and nature of Cooper-like pairing from repulsive interactions; this is based on a configuration mixing realized by a 
canonical transformation; the vacuum is the ground state of the system at $U=0^{+}$and the Cooper-like bound states arise from $W=0$ pairs. Thus our approach is actually not a perturbation theory but its application is easier in weak coupling situations, when one can limit the configuration mixing to a few particle-hole excitations. The pairing mechanism involved is a form of spin-flip exchange diagram which is enhanced by the $C_{4 v}$ symmetry and our canonical transformation works independenly of the size of the system. We have tested the results with exact diagonalization data for open-boundary condition clusters [3] [17] in the three-band Hubbard model. We obtained good agreement with the numerical data already by the simplest approximation within our scheme, i.e. by truncating the configuration mixing at the level of electron-hole pairs exchange.

In this paper we test our $W=0$ pairing mechanism within the one-band Hubbard model with periodic boundary condition using exact numerical data on the $4 \times 4$ square lattice [2]. In particular, several workers have recognised that those data could be qualitatively understood by a weak coupling analysis [11] [12]; however there was no clear-cut conclusion about the existence of pairing and the symmetry analysis of the system was not complete.

We point out the criteria that allow one to unambiguously diagnose pairing of two holes added to the system. At the heart of the effective interaction there is the diagram of Figure 1. To characterize the symmetry of the ground state with 2 added holes we obtained the analytic ground state at half filling for $U=0^{+}$by a new approach [21]. Moreover, we need the symmetry of the $W=0$ pairs in the system at hand. To this end we prove a general result that we call $W=0$ Theorem. The full exploitation of this theorem requires the knowledge of the Optimal Group, that is, a symmetry group which is so big that no accidental degeneracies arise. We have obtained this Group here for the first time. An adequate knowledge of the symmetry of the system allowed us to develop in detail the canonical trasformation on the $4 \times 4$ cluster analytically; thus we obtained good agreement with the data and a clear diagnosis of pairing. This result lends further support to the general approach [15], [16] which predicts pairing in the $N \times N$ cluster for any $N$; moreover, we stress that we have already shown how the the $W=0$ pairing grants the superconducting flux quantization [17].

\section{ACKNOWLEDGEMENTS}

This work was supported by the Istituto Nazionale di Fisica della Materia.

\section{APPENDIX A: CONTRIBUTIONS TO THE $W$ MATRIX FROM FILLED SHELLS}

The $N^{2}$-body determinantal wave functions with $S_{z}=0$ that one can build using the orbitals with $\epsilon(k)<0$ and half of those with $\epsilon(k)=0$ are a set of $\left(\begin{array}{c}2 N-2 \\ N-1\end{array}\right)^{2}$ elements. Each represents one of the degenerate unperturbed $(U=0)$ ground state configurations at half filling. First-order perturbation theory requires the diagonalization of the $W$ matrix over such a basis.

The diagonal elements of the $W$ matrix are just expectation values over determinants $\left|k_{\alpha} \uparrow k_{\beta} \downarrow \ldots\right\rangle$. Such an expectation value is a sum over all the possible pairs of the bielectronic elements of $W$ like

$$
W(\alpha \beta, \alpha \beta)=\sum_{r} U\left\langle k_{\alpha}\left|n_{r}\right| k_{\alpha}\right\rangle\left\langle k_{\beta}\left|n_{r}\right| k_{\beta}\right\rangle=\sum_{r} U \frac{1}{N^{2}} e^{i\left(k_{\alpha}-k_{\alpha}\right) r} \frac{1}{N^{2}} e^{i\left(k_{\beta}-k_{\beta}\right) r}=\frac{U}{N^{4}} N^{2}=\frac{U}{N^{2}}
$$

the result is independent of $k_{\alpha}$ and $k_{\beta}$. Since in any determinant of the set $N^{2} / 2$ plane wave states are occupied for each spin, there are $N^{4} / 4$ pairs, and the diagonal elements are all equal to $U N^{2} / 4$. Thus, the diagonal elements shift all the eigenvalues by this fixed amount.

The off-diagonal elements of the $W$ matrix between determinants that differ by three or more spinorbitals vanish because $W$ is a two-body operator. The off-diagonal elements between determinants that differ by one spin-orbital are sum of contributions like $W(\alpha \beta, \gamma \beta)=\sum_{r} U\left\langle k_{\alpha}\left|n_{r}\right| k_{\gamma}\right\rangle\left\langle k_{\beta}\left|n_{r}\right| k_{\beta}\right\rangle$ that vanish because of the orthogonality of the plane-wave orbitals. One is left with the off-diagonal elements between determinants that differ by two spin-orbitals, which coincide with the corresponding bielectronic elements $W(\alpha \beta, \gamma \delta)=\sum_{r} U\left\langle k_{\alpha}\left|n_{r}\right| k_{\gamma}\right\rangle\left\langle k_{\beta}\left|n_{r}\right| k_{\delta}\right\rangle$. This is just the matrix of $W$ over the truncated Hilbert space $\mathcal{H}$ spanned by the states of the holes in the half filled shell, ignoring the filled 
ones. We stress that there are $N-1$ holes of each spin in $\mathcal{S}_{h f}$, thus $\mathcal{H}$ is much smaller than the full Hilbert space of the Hubbard Hamiltonian; however, since the number of holes grows linearly with $N$, the problem is still far from trivial.

REFERENCES

[1] J. G. Bednorz and K. A. Muller, Z. Phys. B64, 188 (1986).

[2] G. Fano, F. Ortolani and A. Parola, Phys. Rev. B42, 6877 (1990).

[3] Michele Cini and Adalberto Balzarotti, Phys. Rev. B56, 14711 (1997).

[4] W. Fettes and I. Morgensten, Eur. Phys. B9, 635 (1999).

[5] P. Monthoux and D. J. Scalapino, Phys. Rev. Lett. 72, 1874 (1994).

[6] D. Zanchi and H. J. Schulz, Phys. Rev. B54, 9509 (1996).

[7] C. J. Halboth and W. Metzner, cond-mat/9908471 (1999) and Phys. Rev. B 61, 7364 (2000).

[8] P. W. Anderson, Phys. Rev. 115, 2 (1959)

[9] M.C. Gutzwiller, Phys. Rev. Lett. 10, 159 (1963).

[10] W. Metzner and D. Vollhardt, Phys. Rev B 39, 4462 (1989).

[11] B.Friedman, Europhysics Letters 14, 495 (1991)

[12] J. Galan and J. A. Verges, Phys. Rev. B bf 44, 10093 (1991)

[13] $C_{4 v}$ is the symmetry group of a square. It is a finite group of order 8 and it contains 4 one dimensional irreps, $A_{1}, A_{2}, B_{1}, B_{2}$, and 1 two-dimensional one called $E$. The table of characters is

\begin{tabular}{|c|c|c|c|c|c|}
\hline$C_{4 v}$ & $\mathbf{1}$ & $C_{2}$ & $C_{4}^{(+)}, C_{4}^{(-)}$ & $\sigma_{x}, \sigma_{y}$ & $\sigma_{+}^{\prime}, \sigma_{-}^{\prime}$ \\
\hline$A_{1}$ & 1 & 1 & 1 & 1 & 1 \\
\hline$A_{2}$ & 1 & 1 & 1 & -1 & -1 \\
\hline$B_{1}$ & 1 & 1 & -1 & 1 & -1 \\
\hline$B_{2}$ & 1 & 1 & -1 & -1 & 1 \\
\hline$E$ & 2 & -2 & 0 & 0 & 0 \\
\hline
\end{tabular}

[14] Morton Hamermesh, Group Theory and its application to Physical Problems, Addison-Wesley (reading, 1962).

[15] Michele Cini, Gianluca Stefanucci and A. Balzarotti, Solid State Communication 109, 229 (1999).

[16] Michele Cini, Gianluca Stefanucci and Adalberto Balzarotti, Eur. Phys. J. B10, 293 (1999).

[17] Michele Cini, Adalberto Balzarotti and Gianluca Stefanucci, Eur. Phys. J. B14, 269 (2000).

[18] E. H. Lieb, Phys. Rev. Lett. 621201 (1989).

[19] Michele Cini, Adalberto Balzarotti, Raffaella Brunetti, Maria Gimelli and Gianluca Stefanucci, International J. of Modern Physics, in press.

[20] Equivalently, we shall find the exact 6-body ground state of the effective hamiltonian

$$
H_{e f f}=\frac{U}{4^{4}} \sum_{k_{1}, k_{2}, k_{3}, k_{4} \in \mathcal{S}_{h f}} \delta\left(k_{1}+k_{2}-k_{3}-k_{4}\right) c_{k_{1} \uparrow}^{\dagger} c_{k_{2} \downarrow}^{\dagger} c_{k_{3} \downarrow} c_{k_{4} \uparrow}
$$

where $\delta(G)=1$ if $G$ is a reciprocal lattice vector and zero otherwise.

[21] Michele Cini and Gianluca Stefanucci, cond-mat/0009058 and J. Phys.:Condens. Matter 13, 1279 (2001).

[22] A. Moreo and E. Dagotto, Phys. Rev. B41, 9488 (1990). 